E.L.U.A. 3, 1985-1986, págs. 165-192

\title{
TIPOLOGÍA DE LA CATÁFORA PARATÁCTICA: ENTRE LA SINTAXIS DEL DISCURSO Y LA SINTAXIS DE LA ORACIÓN *
}

\author{
JUAN CARLOS MORENO CABRERA \\ (Universidad Autónoma de Madrid)
}

\section{Discurso y sintaxis}

Uno de los desarrollos más recientes de la lingüística de base funcional se fundamenta en el reconocimiento de que no se puede estudiar la sintaxis de la oración aislándola totalmente del proceso textual que se plasma en el discurso. Este aislamiento -que a veces cristaliza en propuestas metodológicas como la tan cacareada de la autonomía de la sintaxis - puede llevarnos a eliminar de nuestro aparato teórico nociones fundamentales para el entendimiento correcto de la sintaxis oracional. En este sentido, no podemos renunciar a tomar en consideración los recientes avances en lingüistica textual ', que van poniendo

* Varias personas me han ayudado de una forma u otra en la elaboración de este trabajo. Entre ellas debo mencionar al Dr. José Polo y al Dr. Javier Elvira quienes me proporcionaron importantes referencias bibliográficas; al Dr. Don Luis Garcia Iglesias, que me proporcionó uno de los ejemplos del griego antiguo; a Ursula Ojanen, que me proporcionó los ejemplos del finés, a Maite Echenique y a Patxi Goenaga cuyas interesantes observaciones sobre el euskera no he sabido aprovechar en este trabajo. Por último debo agradecer a los Dres. Ignacio Bosque y Antonio Garcia Berrio el haberme animado a escribir este articulo.

1 La lingüística del texto se ha ocupado con insistencia de la investigación de los mecanismos de coherencia textual, que son, a nuestro juicio, absolutamente esenciales para estudiar determinados aspectos de la sintaxis oracional. Véase, por ejemplo, E. Bernárdez (1982; cap. 4); T. A. van Dijk (1980; cap. IV); M.A.K. Halliday, R. Hasan (1976), entre otras muchas referencias. 
at descubierto los diversos mecanismos de cohesion discursiva, a la hora de abordar el estudio de la sintaxis. Los estudios de lingüistas como S. Kuno - y T. Givón 'ponen de manifiesto cada vez con más claridad que no existe una ruptura entre la sintaxis del discurso y la de la oracion y que, por tanto, no está justıficada la fractura metodologica ...tipıca de los generativistas - que se concreta en teorias como la ya mencionada de la autonomía de la sintaxis.

\section{Formas de cohesion textual: el pronombre cataforico}

\subsection{Introducción}

Buena parte de la investigación en linguistica textual se ha dedicado, como hemos dicho, al establecimiento y estudio de los medios de cohesión textual; medios estrictamente necesarios para obtener un discurso coherente. M.A.K. Halliday y R. Hasan ${ }^{4}$ han enumerado y estudiado los siguientes procedimientos de cohesión textual: referencia, sustitución, elipsis, conjunción y cohesión léxica. Aqui nos interesa fundamentalmente el primero de ellos, que los autores definen del sIguiente modo:

«What characterizes this particular type of cohesion, that which we are calling REFERENCE, is the specific nature of the information that is signalled for retrival. In the case of reference, the information, the information to be retrieved is the referential meaning, the identity of the particular thing or class of things that is being referred to; and the cohesion lies in the continuity of reference, whereby the same thing enters into the discourse a second time». 5

2 Vèase, por ejemplo, S. Kuno (1975 y 1976), además de otros muchos artículos, en los que este lingüista aprovecha lo mejor de la teoria praguense de la Functional Sentence Perspective. Puede consultarse igualmente T. Givón (1979; cap. V), E. Garcia (1979), D. M. Levy (1979), G. Sankoff, P. Brown (1976); P. J. Hopper, S. A. Thompson (1980); P. Hopper (1982), W. Foley, R. D. van Valin (1984; cap. 7).

3 Véanse las referencias de la nota anterior.

4 M.A.K. Halliday. R. Hasan (1976).

5 M. A. Halliday, R. Hasan (1976, p. 31). Dentro de la escuela de H. Seiler, se considera que otro fenómeno morfológico pone de manifiesto esa continuidad referencial de que hablan M.A.K. Halliday y R. Hasan. Se trata de la concordancia, técnica lingüistica que se halla dentro de la dimensión operacional de la APREHENSION. Véase, por ejemplo, Ch. Lehmann (1982) y M. Ostrowski (1982). Al parecer, existe una conexión diacrónica entre los sistemas de coherencia textual referenciales y el fenómeno de la concordancia; con ello. la 
La referencia catafórica es mencionada explícitamente por los autores $^{6}$. A propósito de ella, aportan un ejemplo inglés que implica el pronombre it «ello» y que es de especial interés para nosotros. El ejemplo en cuestión lo mostramos en (1).

(1) I would never have believed it. They've accepted the whole scheme.

'Nunca to hubiera creído. Han aceptado el proyecto en su totalidad'.

En (1), identificamos el it como una endófora; es decir, como una referencia al propio texto en el que se encuentra ese elemento pronominal. Concretamente, a la oración que le sigue inmediatamente. Los autores se inclinan a considerar esta palabra más como un demostrativo que como un pronombre puro. La evidencia interlingüistica que aduciremos en las secciones subsiguientes de este artículo, mostrará a las claras lo acertado de esta sugerencia.

\subsection{Catáfora interoracional e intraoracional}

Como ejemplo de catáfora interoracional o discursiva, puede servir el texto inglés de (1). En (2) y (3) vemos dos casos de catáfora interoracional en español.

(2) La serpiente estaba detrás de él. Pedro, sin embargo, no se asustó.

(3) Esto es lo que quiere. Nadar y guardar la ropa.

En (2), el y Pedro pueden referirse a la misma persona; en (3) esto se identifica con nadar y guardar la ropa. En ambos casos, el pronombre precede a su "antecedente»: aparece en una oración anterior a la que contiene éste. Por esta razón, podemos hablar en (1), (2) y (3), de catáfora.

También es posible la catáfora intraoracional, tal como lo demuestran los ejemplos de (4) y (5).

(4) Detrás de él, Pedro vio a un niño.

concordancia no vendría a ser otra cosa que el reflejo intraoracional de un proceso interoracional de coherencia textual. Véase, por ejemplo, T. Givón (1976).

6 M.A.K. Halliday, R. Hasan (1976; p. 56). 
(5) En su diario, Juan encontró un billete.

En (4) y (5), él y Pedro son correferentes, al igual que su y Juan; ambas expresiones pronominales preceden a su antecedente en la oración $y$, por tanto, estamos ante dos catáforas intraoracionales ?

Una de las teorias del presente artículo es que existe una transición gradual entre la catáfora interoracional y la catáfora intraoracional. Más adelante, veremos casos intermedios entre ambas en los que se utiliza un pronombre catafórico para anunciar una oración siguiente que, sin embargo, se comporta como proposición subordinada a la proposición principal, en que se halla dicho pronombre. Es decir, casos en los que se cruzan dos procedimientos de relacionar una oración con otra: uno discursivo, consistente en la utilización de un pronombre catafórico como en (1), (2) y (3); y otro de sintaxis intraoracional: la subordinación mediante una conjunción.

También mantendremos que la parataxis está más orientada al discurso que la hipotaxis. Adoptaremos un punto de vista muy amplio sobre la parataxis. Según él, interpretamos etimológicamente ese vocablo gramatical como designador de un proceso de contigüidad discursiva interoracional ejemplificado por (1), (2) y (3). De esta concepción se deduce inmediatamente, según lo que acabamos de exponer, que existe una transición gradual entre la parataxis discursiva y la hipotaxis intraoracional ${ }^{8}$; 0 , si se quiere, entre una loose syntax y una tight syntax. ${ }^{9}$

\section{De la sintaxis paratáctica a la sintaxis hipotáctica}

Tal como sugiere T. Givón ${ }^{10}$, existe una tendencia ontogenética a la adquisición temprana de estructuras sintácticas a medio camino entre

7 Las condiciones que determinan la posibilidad de la catáfora y anáfora intraoracionales han sido objeto de numerosísimos estudios. Podemos citar, entre otros muchos, las siguientes visiones de conjunto sobre el problema: T. Reinhart (1976 y 1983), J. Guéron (1979), Th. Wasow (1979), G. Fauconnier (1974), G. Rigau i Oliver (1981; cap. 5). Para una perspectiva discursiva de la anáfora véase este último libro, páginas 457-80 y además $K$. Stenning (1978). Para una crítica de las teorias intraoracionales desde la perspectiva del discurso véase D. Bolinger (1979).

8 Adoptamos, pues, el método escalar típico de la escuela de $H$. Seiler.

9 Estas expresiones son de T. Givón (vid. T. Givón 1979, p. 208). Recordemos la propuesta de Badia Margarit (1960) de distinción entre una sintaxis suelta y una sintaxis trabada.

10 T. Givơn (1979; pp. 75 ss.). 
el discurso y la oración, que conectan un tema con un predicado a través de un pronombre correferencial con ese tema. La adquisición de estructuras más gramaticalizadas en las que el tema se une directamente al predicado y pasa a ser sujeto, es posterior y obedece a un proceso de condensación sintáctica que, según Givón, posee justificación filogenética. El primer tipo de estructura es ejemplificado por nuestro autor mediante la expresión de (6a), el segundo, mediante (6b).

\begin{tabular}{|c|c|c|c|}
\hline b) a. & $\begin{array}{l}\text { Joe } \\
\text { 'Joe }\end{array}$ & $\begin{array}{l}\text {, he } \\
\text {, él }\end{array}$ & $\begin{array}{l}\text { is sick 'I } \\
\text { está enfermo' }\end{array}$ \\
\hline & TEMA & $\begin{array}{l}\text { PRON. } \\
\text { SUJ. }\end{array}$ & PREDICADO \\
\hline b. & Joe & is sick & \\
\hline & $\begin{array}{l}\text { SUJ. } \\
\text { Joe es }\end{array}$ & $\begin{array}{l}\text { PRED } \\
\text { enfern }\end{array}$ & DO \\
\hline
\end{tabular}

Esta prioridad ontogenética de la construcción TEMA + PRONOMBRE + PREDICADO sobre la más condensada SUJETO + PREDICADO, se corresponde, como hemos dicho, con la prioridad filogenética que se demuestra mediante la evolución diacrónica de estructuras como (6a) a estructuras como (6b) en las más diversas lenguas. Así, según estableció el propio T. Givón ${ }^{12}$, se produce un proceso de reanálisis ${ }^{13}$ que se puede esquematizar como en (7).

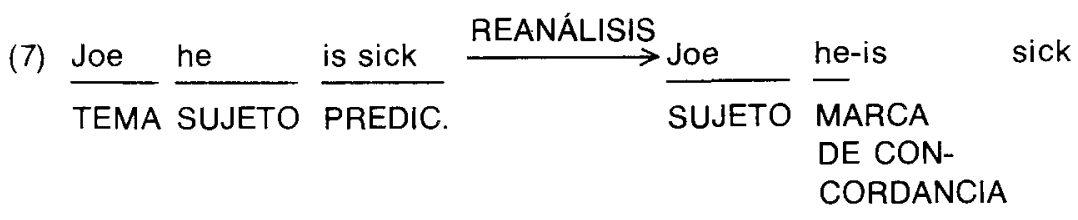

Es decir, cuando el tema pasa a sujeto, el pronombre sujeto se reanaliza como un marcador morfológico de concordancia con el su-

11 L. Taimy (1978) pone en contacto este tipo de estructuras con un proceso sintáctico más general que él denomina copy-clefting; hemos de recordar en este sentido la distinción de Bally entre frase ligada y frase segmentada epifórica y anafórica; véase $H$. Frei (1980).

12 T. Givón (1976).

13 Sobre el reanálisis o reestructuración como mecanismo de evolución sintáctica, véase T. Givón (1971), R. Langacker (1977) y A. Timberlake (1977). Una visión de conjunto, en J. C. Moreno (1978); sección 4.3.4. 
jeto. Este proceso se puede observar, por ejemplo, en la lengua bantú kimbundu ${ }^{14}$. En (8a) tenemos una estructura del tipo (6a) que da origen a (8b), que es del tipo (6b).
(8) a. Nzua , a - mu - mono Juan ellos - a él - vieron
b. Nzua a - mu - mono (kwa meme) Juan fue visto (por mí).

En (8b) Nzua pasa dentro de la oración -es decir, pasa de tema a sujeto-y esto hace que el pronombre sujeto de (8a) a 'ellos', se reanalice como un morfema pasivo, lo que posibilita la aparición del complemento agente kwa meme 'por mi'.

$K$. Lambrecht ${ }^{15}$ nos propone las expresiones (9) del francés no estándar, que ponen de manifiesto con toda claridad el proceso diacrónico visto en el kimbundu.

$$
\begin{aligned}
& \text { (9) a. Moi je-mange et toi tu-bois } \\
& \text { Yo yo-como y tú tu-bebes. } \\
& \text { b. Pierre il-mange } \\
& \text { Pedro él-come } \\
& \text { c. Mes amis ils-boivent } \\
& \text { Mis amigos ellos-beben. }
\end{aligned}
$$

Entre los muchos ejemplos de condensación sintáctica por reanálisis que T. Givón cita ${ }^{16}$, nos interesa especialmente uno. Se trata de la evolución de las subordinadas completivas en hebreo biblico. Es el caso de la condensación sintáctica de un modelo de subordinación más cercano a la parataxis, en otro, de carácter más hipotáctico. Se parte del hecho de que en hebreo bíblico verbos como ver, pensar, saber pueden tomar, como en español, un sintagma nominal objeto o bien una subordinada sustantiva. Existe una construcción en la que el complemento oracional es anticipado catafóricamente por un pronombre, tal como vemos en (10). Podemos denominar tal pronombre como catáfora paratáctica.

\footnotetext{
14 T. Givón (1979, p. 211).

15 K. Lambrecht (1980).

16 T. Givón (1979; pp. 207-33).
} 


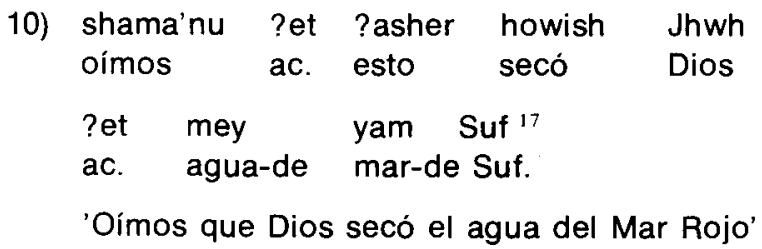

Según T. Givón, a partir de estructuras como la de (10) se desarroIlan otras más trabadas con un verbo y un complemento oracional, como la de (11).
(11) yada'ti she-gam hu? r'ut ruax ${ }^{18}$ supe que también ello vicio espíritu 'Supe que ello también era vicio'.

La oración de (11) incluye una hipotaxis que se desarrolló a partir de una estructura más cercana a la parataxis, como la de (10).

A veces, el cambio ejemplificado no supone alteración ninguna de forma, sino sólo de la estructuración del resultado final. El mismo Givón, siguiendo una sugerencia de Th. Vennemann, cita este caso del inglés: I know that, it is true 'lo sé, es verdad' podría haber evolucionado a I know that it is true 'sé que es verdad'. En el primer caso, that es un pronombre; en el segundo, una conjunción. En este mismo sentido, J. Haudry llama la atención sobre la relación entre anáfora discursiva e hipotaxis relativa:

«Le mécanisme de la réinterpretation est bien connu; il est visible en anglais moderne: entre he came to a river; that was broad and deep et the came to a river that was broad and deep la difference se limite à l'importance plus o moins grande de la pause entre river et that; il y a donc continuité entre l'anaphorique et cet emploi du relatif. Le véritable changement se réalise lorsque l'ancien anaphorique est étendu aux autres fonctions du relatif. » ${ }^{19}$

\section{De la catáfora paratáctica a la hipotaxis mediante el reanálisis}

Como hemos visto anteriormente, vamos a suponer que las estruc-

17 T. Givón (1979; p. 219).

18 ibídem.

19 J. Haudry (1973; p. 173). 
turas de subordinación con catáfora paratáctica son el antecedente de construcciones hipotácticas, en un proceso en el que interviene el fenómeno del reanálisis.

Consideremos la conjunción causal francesa parce que. En francés antiguo, era muy común el giro causal pource que ${ }^{20}$, que muestra un primer estadio en el que comprobamos una catáfora paratáctica como la mostrada en (12).

(12) La femme du marchand l'estimait pour ce qu'il craignait Dieu 21

'La mujer del mercader le estimaba porque él temía a Dios'.

Es decir, estamos ante el esquema de subordinación expuesto en (13).

\section{ESQUEMA DE LA CATÁFORA PARATÁCTICA}

$$
\begin{gathered}
\left(\mathrm{O}\left(\mathrm{P}_{\mathrm{p}} \ldots\left(\text { Prep.) PRON. ...) ( } \mathrm{p}_{\mathrm{s}} \mathrm{CON} J . \ldots\right) \mathrm{O}\right)\right. \\
\text { CATÁfORA PARATACTICA }
\end{gathered}
$$

$\mathrm{P}_{\mathrm{p}}=$ proposición principal

prep. = preposición

PRON. = pronombre

$\mathrm{P}_{\mathrm{s}}=$ proposición subordinada

CONJ. = Conjunción.

En (13) estamos ante una estructura intermedia entre la parataxis y la hipotaxis: el aspecto paratáctico se manifiesta en la catáfora y el hipotáctico, en la presencia de la conjunción subordinante.

En español medieval, la catáfora paratáctica es muy frecuente. En (14) incluimos algunos ejemplos de ella ${ }^{22}$.

(14) a. Por esso vos la do que la bien curiedes.

b. por tal fago aquesto que sirvan a so señor.

c. $\mathrm{Bi}(\mathrm{e})$ ne lo veo sines escarnio, que uno omne es nacido de carne.

Pero en francés se generalizó otro giro causal con la preposición par, es decir, par ce que, que en un momento determinado debió tener

20 Véase, por ejemplo, G. Le Bidois, R. Le Bidois (1971, T. II, p. 450).

21 Le Bidois, Le Bidois (1971, t. II, p. 450).

22 Los ejemplos son de R. Lapesa (1980; p. 218). 
una estructura idéntica a la de pour ce que. Podemos suponer que tuvo lugar un proceso de reanálisis según el cual, primero, ce perdió su cualidad de pronombre y se integró en par como morfema suyo y, luego, la expresión resultante, parce, se metió en la subordinada y la secuencia parce + que se reanalizó como una conjunción. Esta evolución se puede resumir mediante el esquema de (15).

(15)

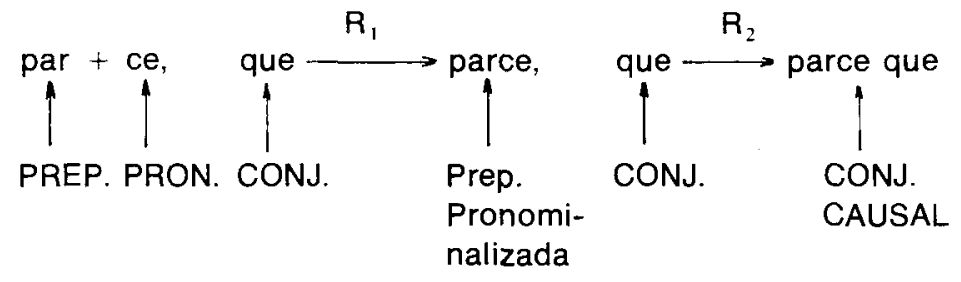

Este paso supone la obtención de estructuras típicamente hipotácticas como la de (16) ${ }^{23}$.

(16) Peu de chose nous console parce que peu de chose nous afflige ${ }^{24}$.

'Pocas cosas nos consuelan porque pocas cosas nos afligen'.

El status de la palabra parce que como conjunción inanalizable, lo vemos en el giro vulgar de (17) ${ }^{25}$.

(17) Parce que quoi qu'il a dit ça?

'¿Por qué ha sido que él ha dicho eso?'

Este reanálisis no se ha producido, en todos los casos en que en

23 Como dice J. Haudry (1973, p. 149) el paso de la parataxis a la hipotaxis es demostrativo de la tendencia a la gramaticalización. Aunque no utiliza la palabra reanálisis, dice respecto de pasos como éste:

'Sans que rien ne change au niveau des significants, la structure de l'énoncé se modifie profondément quand à l'analyse en deux phrases substitue l'analyse en une phrase» $(1973$; p. 150).

Este autor nos advierte, además, de que respecto del latín, la creación de parce que no supone la creación de la subordinación causal ya existente en latín, sino la formación de un mecanismo gramatical que reemplaza las funciones del quia y quod latinos. Véase J. Haudry (1973; p. 148).

24 Le Bidois, Le Bidois (1971; t. II; p. 451).

25 Ibídem, p. 450. 
lugar del pronombre catafórico hay un sustantivo que no se desgasta lo suficiente como para pasar a ser parte de la preposición. Ejemplos de esto son las locuciones a fin que 'a fin de que' y de peur que 'por miedo a que' y similares.

En otros casos, se conserva la catáfora paratáctica en francés moderno, tal como se ve en los ejemplos de (18).

(18) a. J'étais content de ce que le morceau fut assez copieux ${ }^{26}$.

'Estaba muy contento de (esto) que el pedazo fuera tan grande'.

b. Je consens à ce qu'il vienne ${ }^{27}$

'Estoy de acuerdo en que venga'

En español, quizás pueda proponerse un proceso similar mediante el cual se pasa de paráfrasis conjuntivas como por esso que a secuencias de preposición más conjunción como por que. En este proceso habría que establecer una supresión del pronombre y un ulterior reanálisis, tal como mostramos en (19).

(19) por + esso, que $\rightarrow$ por $+\varnothing$, que $\rightarrow$ porque

Obsérvese que en español nunca aparece un pronombre catafórico paratáctico. Así, correspondiendo a las oraciones francesas (18a y b), podriamos suponer (20a y b) como pasos intermedios.

(20) a. Estaba contento de $\varnothing$ que el trozo fuera tan abundante.

b. Estoy de acuerdo con $\varnothing$ que venga.

El español ha desarrollado y extendido la hipotaxis totalmente y ha marginado la construcción de la catáfora paratáctica.

\section{Tipología de la catáfora paratáctica}

A continuación, intentaremos esbozar una tipología de la catáfora paratáctica basándonos en el examen de esta construcción en diferentes lenguas.

26 ibídem, p. 341.

27 Ibídem, p. 341. 


\subsection{Lenguas indoeuropeas}

\subsubsection{Griego antiguo y latín: catáfora paratáctica y correlación pro- nominal paratáctica}

En griego antiguo podemos encontrar ejemplos de catáfora paratáctica como el incluido en (21).

(21) Dià toûto dè ouk eis dódeka phylás synétaxen, hópōs mē symbainēi merídsein kata tàs proûparkhoúsas trittýs ${ }^{28}$.

'No dispuso a los ciudadanos en doce tribus, a fin de evitar dividirlos según los tercios ya existentes'.

La proposición final introducida por la conjunción hópōs es anunciada catafóricamente por la expresión dià toûto 'por esto' que encabeza la oración.

En latín encontramos también casos de catáfora paratáctica tales como los incluidos en (22).

(22) a. In eo est peccatus si non licuit ${ }^{29}$.

'En esto consiste la falta, en que no fue lícito'.

b. Totum in eo est tectorium ut concinnum sit ${ }^{30}$.

'Todo depende de (esto) que el revocado sea bonito'

Las dos oraciones subordinadas están anunciadas catafóricamente por el pronombre de eo.

Una forma de subordinación muy cercana a la parataxis, que se da en latín y en griego, consiste en la introducción de una subordinada adverbial mediante el uso de dos adverbios pronominales correlativos. Este procedimiento deriva del proceso de cohesión discursiva de la repetición -que M. A. K. Halliday y $R$. Hasan incluyen como un caso especial de cohesión léxica " interoracional discursiva se utiliza en la sintaxis intraoracional. En estos casos estamos ante una correlación pronominal paratáctica aún más cercana a la sintaxis discursiva que la catáfora paratáctica.

En latín vuigar, encontramos ejemplos como el de (23).

28 Aristóteles, Constitución de los atenienses, XXI, 1-4.

29 Lisardo Rubio (1983, p. 350).

30 ibidem.

31 M.A.K. Halliday, R. Hasan (1976; pp. 277-82). 
(23) lam autem, quoniam ager publicum erat (...) et ideo iam non fuit necesse vexare milites "

'Pero ahora, como era ya la vía pública (...) ya no era necesario molestar a los soldados'.

Este ejemplo es revelador, porque presenta un claro caso de parataxis - de ahi la conjunción et- en el que se observa la correlación quoniam... ideo que contribuye a establecer un nexo más allá del propiamente paratáctico entre las dos oraciones. Esto muestra que la correlación adverbial paratáctica está muy cerca de la parataxis discursiva.

Veamos otro ejemplo intimamente conectado con el anterior: se trata de (24).

(24) Quia ad plenum discere volebam loca (...) ac sic necesse fuit etiam denuo ad terram Gessen reverti ${ }^{33}$.

'Como queria conocer a fondo los lugares (...) (así) fue necesario volver de nuevo a la tierra de Gesén.'

En este caso, tenemos un cruce entre la hipotaxis y la parataxis, muy revelador, como caso intermedio entre los polos paratácticos e hipotácticos. Tenemos una conjunción subordinante, quia y otra de carácter paratáctico, ac. En este sentido afirma J. Haudry:

«La corrélation se situe à mi-chemin entre la parataxe e l'hypotaxe. Comme dans la parataxe, les deux parties de l'énonciation sont grammaticalement indépendents l'une de l'autre; aucune des deux n'est à considérer comme subordinnée. C'est pourquoi la corrélation s'exprime parfois par des significants identiques mis en parallèle. ${ }^{34}$

En (25) incluimos, por último, un ejemplo típico de correlación adverbial paratáctica.

32 Este ejemplo y los dos siguientes son de la Peregrinatio Aetheriae y nos han sido proporcionados por $V$. Väänänen en una conferencia que pronunció en la Universidad Autónoma de Madrid el 3 de mayo de 1984.

33 Véase nota anterior.

34 J. Haudry (1973; pp. 152-3). De hecho, Haudry sostiene que de las estructuras correlativas surgieron en latín y en otras lenguas indoeuropeas diversas estructuras hipotácticas; entre ellas, la relativa a través del diptico correlativo. Véase para esto Ch. Lehmann (1984, pp. 147 ss., 300 ss.). 
(25) At ubi autem sexta hora se ferecit, sic itur ad crucem. ${ }^{35}$ 'Pero cuando ha llegado la hora sexta, (entonces) va ante la cruz'.

La correlación pronominal paratáctica es muy frecuente en griego desde los himnos homéricos ${ }^{36}$. Como ejemplo de correlación pronominal paratáctica en griego antiguo, podemos señalar el de (26).

(26) Êmos ho xénos throeî taîs aikhmalōtois paisín, têmos thyraíos êlthon hōs hymâs.

'Mientras que nuestro huésped hablaba con los jóvenes cautivos, yo he franqueado la puerta y he venido a buscaros'.

En lenguas no indoeuropeas, como las turcas, también se dan casos en los que una proposición subordinada está señalada en la principal mediante una expresión correlativa. Por ejemplo, podemos citar la oración (27) de la lengua kipchak (del Codex Cumanicus).

(27) ne ücün alar algišlì- dir, aning ücün por que ellos son benditos por eso köktägi khaulig alarning- dłr ${ }^{37}$. el reino de los cielos les pertenece.

Esta misma construcción se da en lenguas modernas como en catalán, tal como indicamos en los ejemplos (28).

(28) a. Quan plovia más fort, aleshores vàrem sortir ${ }^{38}$ 'Cuando llovía más fuerte, entonces salimos'

b. Encara que ho asseguri amb tanta passió, jo tanmateix no m'ho $\mathrm{crec}^{39}$.

'Aunque lo asegure con tanta pasión, yo no obstante, no me lo creo'.

35 Véase nota 32.

36 Véase P. Chantraine (1963, t. Il; pp. 254-55).

37 V. Drimba (1973; p. 166).

38 A. M. Badia Margarit (1962; t. Il; p. 217).

39 Ibídem. 


\subsubsection{La catáfora paratáctica en las lenguas germánicas}

En inglés, se dan también casos de catáfora paratáctica, algunos ejemplos de los cuales, mostramos en (29).

(29) a. They expect it of you that you cooperate ${ }^{40}$. 'Esperan (ello) de ti, que cooperes'.

b. I take it upon myself to fix the lamp ${ }^{4}$.

'Me encargo de poner la lámpara'.

c. It doesn't frighten me to watch horror movies anymo$\mathrm{re}^{42}$.

'(Ello) no me asusta ya ver películas de terror'.

En los tres casos anteriores, el it es un pronombre que anticipa catafóricamente la subordinada. En los tres casos podría prescindirse de la subordinada sin afectar la gramaticalidad de la expresión resultante, que estaria completa gracias al it: asi, they expect it of you 'lo esperan de ti'; I take it upon myself 'me encargo de ello'. En una hipotaxis pura como they said that it was necessary 'dijeron que era necesario', no se puede prescindir de la subordinada sin obtener una expresión agramatical; en este caso they said 'dijeron'. En una estructura con catáfora paratáctica esto no se cumple, tal como hemos visto, y aquí radica precisamente el carácter típico de estas expresiones.

En alemán la catáfora paratáctica es frecuentísima. En (30) vemos algunos ejemplos de la misma.

(30) a. Der friedliche Charakter der Unternehmens schützt jedoch keineswegs davor, das eigene Leben dabei zu riskieren 43.

'El carácter pacífico de la empresa no nos protege sin embargo, en modo alguno, de (esto) arriesgar la propia vida en ella'.

b. Möglichst wenige Informationen darüber, wo die Erntehelfer sind, sollten in der Öffenlichkeit bekannt werden ${ }^{44}$.

J. Emonds (1972; p. 31).

Ibídem.

42 J. Emonds (1972; p. 30).

43 Este ejemplo y el siguiente están extraídos de una conocida revista alemana.

44 Véase la nota anterior. 
'Debe salir a la luz la menos información posible sobre (esto) dónde están los recolectores'.

c. Die Infrarot-Astronomie hat die technischen Voraussetzungen dazu geschaften, dass wir and solchen 'Sterngeburten' beobachtend teilnehmen können ${ }^{45}$.

'La astronomía de infrarrojos ha creado los presupuestos técnicos para (esto) que podamos participar de la observación de tales «nacimiento de estrellas»'.

d. Wir dürfen nicht davon gehen, dass die Wolke ursprünglich überall diesselbe Dicht hat ${ }^{46}$.

'No debemos partir de (esto) que las nubes originariamente tienen en todos los sitios la misma densidad.

e. Bei den zwei ersten handelt es sich darum, dass sich in Innern des Protosternes jeweils ein ruhender hydrostatischer Kern bildet ${ }^{4}$.

'En los dos primeros se trata de (esto) que en el interior de la protoestrella siempre se forma un núcleo hidrostático inerte.'

En todos los casos anteriores, tenemos una expresión pronominal en la proposición principal compuesta de un elemento de carácter deíctico; da, más una preposición: davor 'ante esto', darüber 'sobre esto', dazu 'a esto', davon 'de esto', darum 'alrededor de esto'. Estas expresiones pronominales anuncian la subordinada; es decir, se refieren catafóricamente a ellas.

Frente a esto, existen subordinadas con hipotaxis sin ningún tipo de catáfora paratáctica, como muestran los ejemplos de (31).

(31) a. Wir hoffen, dass du uns bald wieder besuchst.

'Esperamos que pronto nos visitarás de nuevo'.

b. Nur die Haustiere bleiben, weil sie den Mensch gebunden sind.

'Sólo se quedan los animales domésticos, porque están unidos al hombre'.

Una subordinada encabezada por weil puede ser anunciada catafóricamente por una expresión pronominal, como vemos en (32).

45 Ejemplo de W. Stegmüller (1979; t. II, p. 528).

46 W. Stegmüller (1979; t. II, p. 530).

47 W. Stegmüller (1979; t. II; p. 531). 
(32) Ich bleibe heute deshalb zu Hause, weil ich einen wichtigen Anruf erwarte ${ }^{48}$.

'Me quedo en casa, porque espero una llamada importante'.

En alemán, por tanto, coexisten los dos medios de relación intraoracional: la catáfora paratáctica y la hipotaxis pura.

En otras lenguas germánicas como el sueco, encontramos una situación similar al alemán; el ejemplo de (33) es del sueco.

(33) Erik är på gott humör därför att Eva inte har gått ifrån honom. ${ }^{49}$

'Erik está de buen humor por (esto) que Eva no le ha dejado'.

En donde där es un demostrativo, för es una preposición y att, la conjunción.

\subsubsection{Ruso}

En ruso, la catáfora paratáctica es tan común como en alemán. En esta lengua, tenemos como catáfora paratáctica un pronombre to 'aquello' con o sin preposición y una subordinada encabezada por la conjunción chto 'que', pronunciada shto. Un primer ejemplo es el de (34).

(34) Vsë-takí opyt privél meniá $k$ tomú, chto ia ubedilas' v neobjodímosti deïstvovat' na deteï strajami ${ }^{50}$.

'De todas formas, la experiencia me llevó a convencerme de la necesidad de servirme del miedo ante los niños'.

En (34), $k$ es la preposición a, hacia y tomú es la forma dativa de to.

En (35) y (36) incluimos otros dos ejemplos más.

(35) Ėti slové ne tol'ko ubedili meniá $v$ tom, chto ia ne krasavets... ${ }^{51}$

48 Los ejemplos (31 a, b) y (32) están extraídos de D. Schulz, H. Griesbach (1982), de las páginas 289 y 315 , respectivamente.

49 L. G. Andersson (1973; p. 178).

50 El ejemplo es de L. Tolstoi, de su obra Détstvo, Otrochestvo, lunost. La transcripción ha sido realizada de acuerdo con los criterios establecidos en J. Calonge, 1969.

51 Ejemplo de L. Tolstoi; véase nota anterior. 
'Estas palabras no sólo me convencieron de (esto) que no soy una belleza...'

(36) la zlilas' ot togó, chto ne ponimala egó ${ }^{52}$ 'Sollocé porque no le entendía'.

En (35) $v$ es la preposición en y tom es el caso prepositivo de to. En (36) ot es la preposición de, desde y togó es el genitivo de to.

En ruso, se ha dado un proceso similar al descrito antes para la conjunción francesa parce que ${ }^{53}$, respecto de la perifrasis conjuntiva potomú, chto, que vemos en el ejemplo de (37).

(37) My ne poéjali zá gorod potomú, chto shël dozhd' 'No salimos al campo por (esto) que llovía.'

Evidentemente, potomú procede de la secuencia po, una preposición rusa que exige dativo, y tomú, dativo de to, tal como parce procede de par y ce. Pero el ruso no continúa este proceso como el francés, pues la conjunción no es incorporada a esta palabra: potomú, chto. En ruso se ha cristalizado ese estado intermedio por el que debió pasar la conjunción francesa parce que.

El ruso emplea también la catáfora paratáctica cuando una oración es el complemento de un sustantivo, adjetivo o adverbio. En (38 a y b) vemos dos casos de catáfora paratáctica que anuncia el complemento proposicional de un adverbio — -38 a) - y de una locución adverbial -(38 b)-.

(38) a. Nezavísimo ot togó, vjodiat oní v voennye soyuzy... 'Independientemente de (esto) si establecen alianzas bélicas...'

b. V mesto togó, chtoby s nami razgovarivat', ty vsë vremia chitáesh gazetu.

'En vez de charlar con nosotros, te pasas el tiempo leyendo el periódico'.

En otras lenguas eslavas no tiene lugar esta catáfora paratáctica; asi, en polaco, la oración ( 38 b) se traduciría como señalamos en (39).

(39) Zamiast rozmawiac $z$ nami, caly czas czytasz gazete ${ }^{54}$. misma traducción que $(38 \mathrm{~b})$.

52 Ejemplo de L. Tolstoi; véase la nota 50.

53 Véase la sección 4 del presente artículo.

54 S. Karolak, D. Wasilewska (1977; p. 50). 
En (39), zamiast rozmawiac, literalmente, 'en vez de hablar' contrasta con el ruso $V$ mesto togó, chtoby razgovárivat, literalmente, 'en vez de esto que charlar'. El español adopta una solución intermedia entre ambas: «en vez de charlar».

En alemán, la catáfora paratáctica es también muy frecuente en estos casos, tal como muestra la expresión unabhängig davon, dass 'independientemente de (esto) que'.

\subsection{Lenguas no indoeuropeas}

\subsubsection{Finés}

En finés son también frecuentes los casos de catáfora paratáctica. Podemos citar los ejemplos de (40).

(40) a. Hän tuli tänne, siksi että hän tapaisi él vino aquí por eso que él encontrará sinut. a ti 'Él vino aquí para encontrarse contigo'

b. Eeva pääsi mukaan sen tähden, että hän tunsi tien A Eva llevaron consigo esto por que ella conocía el camino.

'Llevaron a Eva consigo porque conocía el camino'

c. Tämä on tarpeellista sen vuoksi, että työ on esto es necesario esto por que trab. es vaarallista. peligroso

'Esto es necesario porque el trabajo es peligroso'

La proposición subordinada es anunciada por una expresión compuesta de pronombre más posposición: sen tähden, sen vuoksi o una palabra con una raíz pronominal como siksi. La subordinada es introducida por la conjunción että 'que'.

\subsubsection{Húngaro: Tema, foco y catáfora paratáctica}

El húngaro es quizás la lengua más rica, de entre las que hemos examinado, respecto del fenómeno de la catáfora paratáctica y de la 
correlación pronominal paratáctica. Consideremos, en primer lugar, el ejemplo de (41).

(41) A diák megoldja a feladatát anélkül hogy a tanár el alumno resuelve su tarea ello-sin que el prof. segitene neki ayude a él

'El alumno ha resuelto sus deberes sin que le ayude el profesor'.

Tenemos, pues, en (41), la secuencia anélkül compuesta del pronombre a 'aquello' más nélkül 'sin', que anuncia la subordinada introducida por la conjunción hogy 'que'.

Otro caso similar es el de (42).

(42) Azért ült le, mert elfáradt ${ }^{55}$. aquello-por sentó porque cansó 'Se sentó porque se cansó'

Azért está compuesto del pronombre demostrativo az 'aquello' y el sufijo causalis-finalis -ért ${ }^{56}$; mert es la conjunción causal porque.

La catáfora paratáctica se da incluso en casos en los que no se suele encontrar en otros idiomas. Oraciones como las de (43) son similares a la hebrea de (10).

$$
\begin{aligned}
& \text { a. Azt hiszem, hogy igazad van }{ }^{57} \\
& \text { eso }+ \text { ac. creo que tu verdad es } \\
& \text { 'Creo que tienes razón'. }
\end{aligned}
$$

b. Attól félek, hogy megfáztak ${ }^{58}$ eso + de temo que coja frío

'Temo resfriarme'

En estos casos intervienen en húngaro dos factores: la colocación en la oración del tema y el foco de la misma y la tendencia a situar al

55 Ejemplo de Z. Bánhidi et al. (1980; p. 387).

56 Curiosamente, el sufijo húngaro '-ért posee la misma polisemia que la de la preposición española por: un significado causal y otro final; asi lo hizo por vergüenza y vino aquí por ayuda; de ahi el nombre causalis-finalis. Un estudio sobre este sufijo en húngaro y su equivalente en inglés, en B. Korponay (1982).

57 Z. Bánhidi et al (1980; p. 387).

58 lbídem. 
final de la oración los constituyentes más largos y complejos de ella ${ }^{59}$. El foco en la oración húngara ha de situarse inmediatamente delante del verbo: si el foco es una proposición subordinada, habría que situar ésta delante del verbo, contraviniendo el segundo de los factores mencionados. Este conflicto se soluciona poniendo una catáfora en la posición de foco, un elemento de complejidad mínima. Indicamos este proceso en (44).

(44) János azt mondta, hogy

János eso + ac. dijo que

TEMA FOCO

Zsuzsa holnap nem jön ${ }^{60}$.

Zsuzsa mañana no viene

'János dijo (esto) que Zsuzsa no viene mañana'

El tema ha de aparecer en el lugar inicial de la oración y si la subordinada lo es, se recurre de nuevo y por las mismas razones a la catáfora paratáctica, tal como vemos en (45).

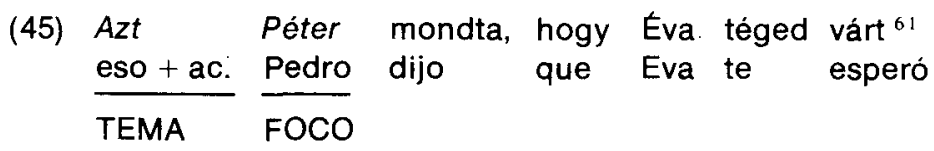

'Pedro dijo que Eva te esperó'

La correlación pronominal paratáctica es muy frecuente en húngaro, tal como podemos comprobar en los ejemplos de (46).
a. Azóta eso + desde nézem meg a magyar nyelvü filmeket veo los húngaro de lengua películas + ac. que + desde bien entiendo húngaro 'Veo películas en lengua húngara, desde que entiendo bien el húngaro'.

59 Tendencia estudiada y formulada recientemente por S. C. Dik (1978, pp. 192 ss.).

60 El ejemplo y el análisis es de C. de Groot (1981 a; p. 46).

61 El ejemplo y el análisis es de C. de Groot (1981 a; p. 47). Son ya muchos los autores que han propuesto este análisis del tema y el foco en húngaro; por ejemplo $\mathrm{C}$. de Groot (1981 a y b). L. Dezsö (1980). K. E. Kiss (1981, 1982), J. Horvath (1976) y J. Andor (1978). 
b. A gyerekek addig

maradtak a szállódában, los niños eso + durante permanecer en el hotel amig a szülök a müsort nézték que + mientras los padres el programa vieron. 'Los niños permanecieron en el hotel mientras que sus padres veían el programa'.

En (46 a y b) tenemos dos expresiones pronominales correlativas que muestran el mismo sufijo: azóta... amióta; addig... amig. La segunda de ellas posee valor de conjunción relativa. Esto hace que no se trate de una correlación paratáctica pura ya que el segundo miembro de la misma está encabezado por un relativo ami constituido por el artículo a más $m i$ 'que'. A los ejemplos de (46) les asignamos el fenómeno de la correlación pronominal relativa, paralela a la correlación pronominal temporal del latín ya vista (cfr. ejemplo (25)). La correlación paratáctica pura presenta dos adverbios correlativos sin que ninguno de ellos sea a su vez conjunción. Por tanto, estos casos están entre la correlación paratáctica pura y la catáfora paratáctica.

\section{Conclusión: de la correlación pronominal paratáctica a la hipota- $\mathrm{xis}$}

\subsection{Escala de la relación interoracional}

A lo largo del presente estudio, hemos ido viendo una serie de construcciones sintácticas que se sitúan entre dos polos opuestos. Uno se corresponde con lo que se podría denominar sintaxis suelta ${ }^{62}$, típica del discurso y resultado de la interacción de los diversos mecanismos de cohesión textual entre los que cabe destacar en este contexto la referencia y la reiteración. Este polo se sitúa pues, en el nivel de la sintaxis del discurso. El otro polo se corresponde con la sintaxis

62 Como ya hemos visto en la nota 9, el término sintaxis suelta es utilizado por A. Badía Margarit (1960), quien lo opone al de sintaxis trabada. Por su parte G. Brettschneider (1980) propone una dimensión de condensación que supone un continuum entre el nivel textual y el de la oración simple:

«... ergibt sich, in einzelsprachlich spezifischer Abstufung von diskreten Konstruktionstypen, ein Kontinuum, das vom Textstück (...) bis hin zum einfachen Satz reicht, in dem eine konzeptuelle Struktur in kondensierter Form as Term, d.h.als Phrase repräsentiert ist. Di Ausprägung dieser einzelnen Abstufungen ist selbstverständlich von Sprache zu Sprache verschieden" (p. 194).

Un buen estudio interlingüistico sobre las relaciones entre parataxis e hipotaxis puede verse en L. Talmy (1978). 
oracional y se cristaliza sobre todo en el fenómeno de la hipotaxis. La transición entre la parataxis y la hipotaxis es la transición entre ambos polos: la primera, más cercana a la sintaxis del discurso, la segunda, integrada en la sintaxis de la oración. La evolución diacrónica de una sintaxis paratáctica a otra hipotáctica, así como la precedencia ontogenética de la primera sobre la segunda es un hecho reconocido ya por los investigadores desde hace tiempo ${ }^{63}$.

Entre ambos polos, se sitúa la catáfora paratáctica, que participa tanto de la parataxis discursiva como de la hipotaxis oracional: se utiliza en ella un procedimiento de cohesión discursiva como el de la referencia y, a la vez, un procedimiento hipotáctico que pone de manifiesto la utilización de la conjunción subordinante. Entre la catáfora paratáctica y la parataxis pura, se sitúa la correlación pronominal paratáctica en la que se utiliza el mecanismo de cohesión discursiva de la reiteración.

El proceso que conduce de la catáfora paratáctica a la hipotaxis pura, mediante el reanálisis, es un resultado del desgaste del pronombre, que va perdiendo progresivamente su valor referencial y convirtiéndose en un mero componente de una conjunción. Es un proceso similar al que describe T. Givón ${ }^{64}$ cuando afirma que en muchas lenguas el morfema de concordancia no es más que un pronombre desgastado.

Una vez examinados los ejemplos de las diversas lenguas estudiadas, podemos establecer una escala con los dos polos de la sintaxis discursiva y sintaxis oracional en la que podemos situar las construcciones estudiadas. Con ello se refieja en la sincronía tipológica un mecanismo de la evolución sintáctica diacrónica ${ }^{65}$.

SINTAXIS SUELTA

SINTAXIS TRABADA

DISCURSO

ORACION

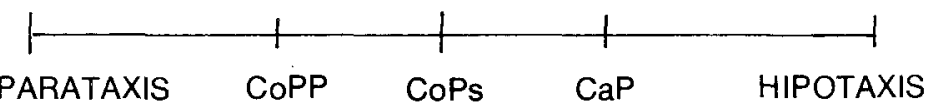

CoPP $=$ Correlación Pronominal Paratáctica

CoPs $=$ Correlación Paratáctica de subordinación causal, final, etc. $\mathrm{CaP}=$ Catáfora paratáctica

63 Véase, por ejemplo, W. von Wartburg (1951; pp. 151-6) y J. Haudry (1973), quien nos advierte, sin embargo, contra el mito de la parataxis primitiva.

64 T. Givón (1976).

65 Sobre las posibilidades que esto abre para el desarrollo de un punto de vista pancrónico, véase J. C. Moreno (1984). 
Este cuadro no pretende reflejar que la hipotaxis surja necesariamente de los pasos mencionados, sino sólo situar las diversas construcciones estudiadas entre los polos de la sintaxis del discurso y de la oración. Todo lo más, el esquema puede reflejar a grandes rasgos una tendencia de evolución que no tiene por qué darse exactamente tal como se especifica en él.

\subsection{La perífrasis de relativo y la catáfora paratáctica}

Como hemos visto, el español moderno no conoce la catáfora paratáctica que, sin embargo, era frecuente en etapas anteriores de nuestra lengua.

Existe una construcción sintáctica muy frecuente entre las lenguas, en la cual se focaliza un elemento de una oración "sacándolo" de ésta y uniéndole de nuevo a ella mediante la cópula. Se trata de lo que los gramáticos de lengua inglesa llaman cleft sentences 'oraciones escindidas'; en español es más conveniente utilizar la expresión perifrasis de relativo, pues no existen cleft sentences en nuestra lengua, sino una construcción parecida, aunque no idéntica ${ }^{66}$. El ejemplo de (47) es una muestra de esta construcción: o, mejor dicho, de una variante suya.

(47) El que vino fue Juan

En (47) el que constituye un elemento unitario, sujeto del verbo vino, como lo prueba el hecho de que puede sustituirse por el relativo quien: quien vino fue Juan ${ }^{67}$. Además en esta construcción la relativa no tiene antecedente.

Si examinamos la traducción al inglés, alemán, ruso y húngaro ${ }^{68} \mathrm{de}$ la perífrasis relativa de (47) nos daremos cuenta de que en los cuatro casos no existe relativa sin antecedente, sino un pronombre modificado por una relativa. En efecto, en (48) incluimos las traducciones anunciadas de (47).

66 Vid. J. C. Moreno (1983).

67 Cabe, según nosotros, otra interpretación en la que el que ES UNA SECUENCIA de pronombre átono más relativo. En este caso ya no estamos ante una perifrasis de relativo sino ante una copulativa cuyo sujeto -el el átono- está determinado por una relativa. La perifrasis de relativo se caracteriza por constar de una relativa sin antecedente. Véase la referencia de la nota anterior.

68 Para un estudio interlingǘstico de las construcciones cleft y pseudo-cleft -las oraciones de (48) son ejemplos de este último tipo-véase H. Harries-Delisle (1978). 


\author{
a. Alemán \\ Derjenige, der kam, war Johannes \\ b. Inglés \\ The one, who came, was John \\ c. Ruso \\ tot, kto priéjal, èto Iván \\ d. Húngaro \\ $A z$, aki jött, István volt
}

En todos los casos, la relativa entre comas tiene como antecedente un sintagma pronominal; esto no ocurre, como hemos visto, en español. Todas estas lenguas conocen en mayor o menor medida la catáfora paratáctica; el español moderno, no. Si suponemos que la construcción española de (47) deriva de otra más antigua en la que el era el pronombre antecedente de la relativa que vino, podríamos poner en contacto esta construcción con la presencia en castellano antiguo de catáforas paratácticas, obteniendo así una situación similar a la del alemán, inglés, ruso y húngaro modernos. El español parece haber ido más lejos que estas lenguas en el proceso de gramaticalización de estructuras discursivas y el hecho de que en nuestra lengua no exista actualmente la catáfora paratáctica ha de relacionarse con la presencia de la construcción perifrástica de relativo que, como vemos, no existe en otras lenguas europeas ${ }^{69}$.

\title{
Referencias bibliográficas
}

J. Andor (1978), "On primary Topicalization", en J. J. Jaeger et al. (eds.) Proceedings of the Fourth Annual Meeting of the Berkeley Linguistic Society. 1978, pp. 18-29.

L. G. Andersson (1973), «Some subordinating constructions in Swedish", en C. Corum et al. You take the high node and l'll take the low node. Chicago, 1973; pp. 178-199.

A. M. Badia Margarit (1960), «Dos tipos de lengua, cara a cara», en Studia Philologica. Homenaje ofrecido a Dámaso Alonso. Madrid, 1960. T. I; pp. 115-39.

- (1962), Gramática Catalana, Madrid, 2 vols.

69 Como contrapartida, el inglés y alemán conocen auténticas cleft como, por ejemplo, it is John who.knows it 'es Juan el que lo sabe'. Esta construcción está más cerca de la sintaxis del discurso que la perífrasis de relativo española: el it es una catáfora paratáctica. 
Z. Bánhidi et al. (1980), Learn Hungarian. Budapest.

E. Bernárdez (1982), Introducción de la lingüistica del Texto. Madrid.

G. Le Bodis, R. le Bidois (1971), Syntaxe du Française Moderne, Paris, 2 vols.

D. Bolinger (1979), «Pronouns in Discourse», en T. Givón (ed.) 1979, pp. 289-310.

G. Brettschneider (1980), «Zur Typologie komplexer Sätze:vorüberlegungen», en G. Brettschneider, Ch. Lehmann (hrsg), 1980, pp. 192-98.

G. Brettschneider, Ch. Lehmann (hrsg.) (1980), Wege zur Universalienforschung. Tübingen.

J. Calonge (1969), Transcripción del ruso al español. Madrid.

P. Chantraine (1963), Grammaire Homérique, Paris, 2 vols.

L. Dezső (1980), "Word Order, Theme and Rheme in Hungarian and the Problems of word-order acquisition», en L. Dezső, W. Nemser (eds.), Studies in English and Hungarian Contrastive Linguistics. Budapest, 1980, pp. 245-98.

- (ed.) (1982), Contastive Studies. Hungarian English. Budapest.

T. van Dijk (1980), Texto y Contexto. Madrid.

S. C. Dik (1978), Functional Grammar. Amsterdam (hay versión española).

J. Emonds (1972), «A reformulation of certain syntactic transformations", en S. Peters (ed.), Goals of Linguistic Theory, New Jersey, 1972, pp. 21-62 (hay versión española).

G. Fauconnier (1974), La Coréférence: syntaxe ou sémantique? Paris.

W. Foley, R. V. Valin (1984), Functional Syntax and Universal Grammar., Cambridge.

H. Frei (1980), "Sur la distinction entre phrases segmentées anaphoriques et epiphoriques", en G. Brettschneider, Ch. Lehmann (hrsg.) (1980), pp. 107-109.

E. García (1979), “Discourse without Syntax» en T. Givón (ed.) (1979) pp. 23-50.

T. Givón (1971), «Historical Syntax and Synchronic Morphology: and archaelogist's Field Trip», en D. Adams et al. (eds.) Papers from the Seventh Regional Meeting, Chicago, 1971, pp. 394-415.

- (1976), «Topic, Pronoun and Grammatical Agreement», en Ch, N., Li (ed.) 1976, pp. 149-88.

- (1979), On Understanding Grammar, New York.

- (ed.) (1979) Syntax and Semantics, 12. Discourse and Syntax, New York.

J. Greenberg (ed.) (1978), Universals of Human Language, Stanford, 4 vols. 
C. de Groot (1981a), «Sentence-Intertwining in Hungarian» en A. M. Bolkenstein et al. (eds.), Predication and Expression in Functional Grammar, Londres, 1981, pp. 41-62.

- (1981b), «On theme in Fuctional Grammar. An Application to some constructions in spoken Hungarian». En T. Hoekstra, $H$. van der Hulst, M. Moorgat (eds.) Perspectives on Functional Grammar, Dordrecht, 1981, pp. 75-88.

J. Guéron (1979), «Rélations de Coréférence dans la phrase et dans le discours" en Langue Française n. ${ }^{\circ} 44$, 1979, pp. 42-79.

M.A.K. Halliday, R. Hasan (1976), Cohesion in English, Londres.

H. Harries-Delisle (1978), "Contrastive Emphasis and Cleft Sentences", en J. Greenberg (ed.) 1978, t. IV, pp. 519-86.

J. Haudry (1973), «Parataxe, Hipotaxe et Corrélation dans la phrase latine», Bulletin de la Société de Linguistique de Paris. T. LXVIII, n. ${ }^{\circ} 1$, pp. 147-86.

P. J. Hopper, S. A. Thompson (1980), «Transitivity in Grammar and Discourse" Language, 56, 1980, pp. 251-99.

P. J. Hopper (1982), «Aspect between Discourse and Grammar» en P. J. Hopper (ed.) Tense and Aspect: between semantics and pragmatics. Amsterdam, 1982, pp. 3-18.

J. Horvath (1976), «Focus in Hungarian and the $\bar{x}$ notation», en Linguistic Analysis, vol. 2, n. ${ }^{\circ} 2,1976$, pp. 175-97.

S. Karolak, D. Wasilewska (1977), Uchébnik pol'skogo iazyká, Varsovia.

K. E. Kiss (1981), "Structural Relations in Hungarian, a «free» Word Order Language", en Linguistic Inquiry vol. 12, n. ${ }^{\circ} 2,1981$, pp. 185-213.

- (1982), «Hungarian and English: a topic-focus prominent and a subject prominent laguage», en L. Dezsö (ed.) 1982, pp. 39-60.

B. Korponay (1982), "A double-faced case category", en L. Dezso (ed.) 1982, pp. 61-74.

S. Kuno (1975), «Three Perspectives in the Functional Approach to Syntax» en R. E. Grossman, L. San, T. Vance (eds.) Papers from the parasession on Functionalism, Chicago, 1975, pp. 276-336.

- (1976), «Subject, Theme and the Speaker's Empathy- A reexamination on relativization phenomena». En. Ch. N. Li (ed.) (1976) pp. 417-44.

R. Langacker (1977), «Syntactic Reanalysis» en Ch. N. Li (ed.) 1977, pp. 57-140.

K. Lambrecht (1980), «Topic, French Style. Remarcks about a basic sentence type of Modern Non-Standard French" en B. R. Caron et al. (eds.) Proceedings of the Sixth Annual Meeting of the Berkeley Linguistics Society, Berkeley, 1980, pp. 337-60. 
R. Lapesa (1980), Historia de la Lengua Española, Madrid.

Ch. Lehmann (1982), "Universal and Typological aspects of agreement" en H. Seiler, F. Stachowiak (hrsg.) (1982), pp. 201-67.

- (1984), Der Relativsatz, Tübingen.

D. M. Levy (1979), "Communicative Goals and Strategies» en T. Givón (ed.) 1979 , pp. 183-212.

Ch. N. Li (ed.) (1976), Subject and Topic, New York.

- (1977), Mechanism of Syntactic Change. Texas.

J. C. Moreno (1978), La Lingüistica Histórica Generativa: aproximación sistemática, Memoria de Licenciatura Inédita, Universidad Autónoma de Madrid.

- (1983), «Las perífrasis de relativo», en Serta Philologica F. Lázaro Carreter, Madrid, 1983, t. I, pp. 455-68.

- (1984), «Diacronía y Tipología hacia una superación del punto de vista sincrónico», Universidad Autónoma de Madrid.

M. Ostrowski (1982), «Zum Konzept der Kongruenz» en H. Seiler, Ch. Lehmann (eds.) 1982, pp. 252-70.

T. Reinhart (1976), The Syntactic Domain of Anaphora, Tesis Doctoral M.I.T.

- (1983), Anaphora and Semantic Interpretation, Londres.

G. Rigau i Oliver (1981), Gramàtica del discurs. Barcelona.

L. Rubio (1983), Introducción a la sintaxis estructural del latín, Barcelona.

G. Sankoff, P. Brown (1976), «The Origins of Syntax in Discourse" en Language 52.

D. Schulz, H. Griesbach (1982), Grammatik der deutschen Sprache, Munich.

H. Seiler, Ch. Lehmann (hrsg.) (1982), Apprehension. Das sprachliche Erfassen von Gegenständen. Tel I. Bereich und Ordnung der Phänomene, Tübingen.

H. Seiler, F. Stachowiak (hrsg.) (1982), Apprehension. Das sprachliche Erfassen von Gegenständen. Teil II. Die Techniken und ihr Zusammenhang in Einze/sprachen, Tübingen.

W. Stegmüller (1979), Hauptströmungen der Gegentwartsphilosophie, Stuttgar, 2 vols.

K. Stenning (1978), «Anaphora as an approach to pragmatics» en M. Halle, J. Bresnan, J. A. Miller (eds.) Linguistic Theory and Psychological Reality, Cambridge, 1978, pp. 162-200.

L. Talmy (1978), «Relations between subordination and coordination» en J. Greenberg (ed.) 1978, t. IV, pp. 487-514.

A. Timberlake (1977), «Reanalysis and Actualization in Syntactic Change» en Ch, N. Li (ed.) 1977, pp. 141-254. 
W. von Wartburg (1951), Problemas y Métodos de la Lingüistica. Madrid.

Th. Wasow (1979), Anaphora in Generative Grammar, Gantes. 